\title{
APODIZATION TECHNIQUES FOR SPURIOUS MODE SUPPRESSION IN 900 MHZ ALUMINUM NITRIDE CONTOUR-MODE RESONATORS Marco Giovannini ${ }^{1,2}$, Serkan Yazici ${ }^{1}$, Nai-Kuei Kuo ${ }^{1}$, and Gianluca Piazza ${ }^{3}$ \\ ${ }^{1}$ University of Pennsylvania, Philadelphia, Pennsylvania, USA \\ ${ }^{2}$ Politecnico of Milan, Milan, Italy, and \\ ${ }^{3}$ Carnegie Mellon University, Pittsburgh, Pennsylvania, USA
}

\begin{abstract}
This paper reports, for the first time, on the application of apodization techniques to $900 \mathrm{MHz}$ MEMS AlN Contour-Mode Resonators (CMRs [1]) to efficiently suppress spurious modes in close proximity of the main mechanical resonance. This concept has been applied with excellent results to a variety of one port resonators formed by patterned top electrodes made out of Aluminum, and a floating bottom electrode made out of Platinum sandwiching the AlN film. As also predicted by 3D COMSOL simulations, a complete elimination of spurious responses in the admittance plot of these resonators is attained without impacting their $Q$ or electromechanical coupling coefficient, $k_{t}^{2}$.
\end{abstract}

\section{INTRODUCTION}

The presence of spurious modes hinders the performance of filters by generating ripples in the pass-band and unwanted responses out-of-band. A spurious free response also could help in reducing phase noise in oscillators. Additionally, the ability to set the device impedance by changing its geometry is limited by the appearance of spurious modes for certain device aspect ratios. Although some methods to suppress out-of-band spurious by means of anchoring techniques [2],[3], or the introduction of dummy electrodes [4] have been implemented, the problem of inband spurious suppression for CMRs has never been addressed.

This paper introduces the concept of apodization for MEMS resonators - a new method to shape the geometry of the electrodes in order to obtain a consistent reduction of spurious modes of vibration in the electrical response of one port CMRs.

The concept of apodization consists of confining the vibration energy in specific regions of the CMR body by shaping its electrodes.

To implement the apodization technique, a standard CMR is used as a reference and its electrodes are modified. Two apodization techniques were studied and applied to one port resonators formed by patterned top electrodes $(25,33,49$ and 65 fingers) made out of Aluminium (120 and $220 \mathrm{~nm}$ thick), and a floating bottom electrode made out of Platinum (90 nm thick) sandwiching the AIN film (500 thick) (see Figure1):

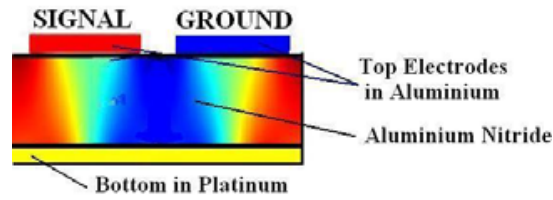

Figure 1. Schematic representation of the finger pair forming the basic element of a lateral field excited CMR.

In the first proposed apodized shape design (shape I, see Figure 2), the electrode overlap progressively decreases when moving from the central part of the resonator towards the edges following a sine like shape. Instead, in the second developed apodized shape (shape II, see Figure 2), the electrode overlap decreases when moving away from the central part of the resonator, but maintains constant for the last set of fingers towards the edges. This variation was implemented with the scope of keeping a larger electrode overlap area, and therefore a higher $k_{t}^{2}$. This concept was applied to a variety of one port CMRs (different geometry and material thickness) in order to prove its effectiveness and repeatability. Each solution has been designed, simulated, fabricated and tested. a)

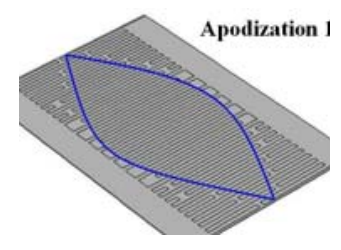

b)

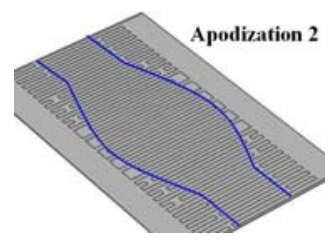

Figure 2. Apodization: shape I (a), shape II (b).

\section{APODIZATION THEORY}

Apodization techniques were first introduced with surface acoustic wave (SAW) transducers in order to improve the sampling of the signal coming from an unapodized SAW device. This technique is the most widely used method for weighting the response of a SAW transducer and pick up exclusively the desired modes of vibration. It relays on properly varying the shape of the electrodes so that the sampled electric signal is properly weighted (Fig. 3) by a prescribed window (sampling) function.

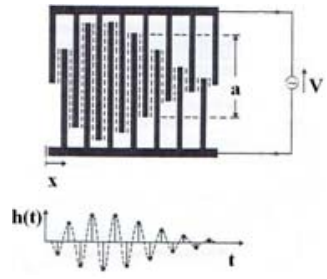

$\mathrm{V}$ : Electric potential a : Overlap electrode length $\mathrm{h}(\mathrm{t})$ : Impulse response

Figure 3. Schematic representation of the apodization technique and its equivalent impulse response.

Although the CMR does not excite a surface acoustic wave, but a lamb wave, the device is formed by a large set of interdigitated electrodes and the same concept of apodization can be implemented to suppress transversal vibrations that cause undesired spurious vibrations in standard, unweighted CMRs. As for SAWs, in the CMRs, the electric field is applied to the AIN body through a set of interdigitated electrodes (also known as fingers). So, shaping of the CMR fingers permits to accurately weigh the electric field distribution that is applied to the CMR. Effectively, the apodization concept consists of finding a method to shape the electrodes so as the electric field is applied in a manner that exclusively the main vibration mode of interest is excited. Apodization techniques are based on the idea that it exists a relationship between the "impulse response" of the device acoustic waveform, the electrodes geometry and its frequency response [5]. Because of this relationship, (due to the nature of the piezoelectric actuation), the device impulse response is seen as a sequence of Dirac functions at times corresponding to the electrode locations, with amplitudes given by the electrode overlap length, $a$ (Fig. 3). The idea of apodization consists in placing and shaping 
the electrodes at those locations corresponding to the peaks and thorough of the waveform (that is propagating) so as to excite the desired mode of vibration. This means that we will maximize the electrode overlap $(a)$ at those locations where the wave amplitude is maximum and shape the electrodes so as to follow the desired frequency response that we want to obtain. Thus, the point is to shape the CMR electrodes layout so as the device impulse response follows the desired frequency response. In the standard case, the impulse response is composed by a series of completely overlapped electrodes. In the case of a 13 fingers one port CMRs, we can identify the electrode layout with a vector (Fig. 4), where the unit number, positive or negative, represents the fact that the electrode (signal or ground) extends over the entire length of the device. The + or - sign is used to distinguish whether the electrode is a signal or ground electrode. A zero represents that no electrode is present in that region (generally in between electrode pairs).

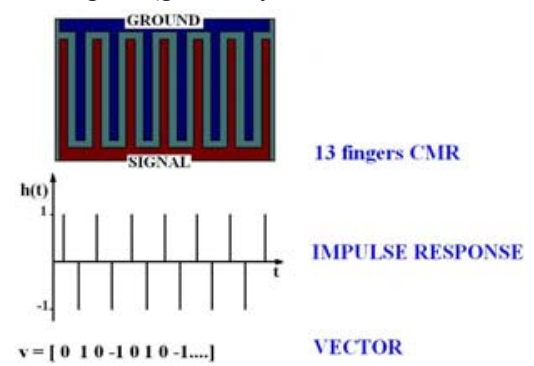

Figure 4. Figure of 13 fingers CMRs with its impulse response and associated vector.

Assuming that this distribution corresponds to the impulse response in time of the resonator, its frequency response can be found by a simple Fourier transform of this vector (Fig.6). It is evident that, in this case, the CMR response is characterized by a relatively spread out lobe for the main frequency, plus other smaller adjacent lobes. Clearly, the energy is not fully confined in a single frequency.

Instead, when we apply apodization to the same CMR, and we adopt, for example, the sine shape window for the electrode design, the same vector (Fig. 4) can be rewritten (Fig.5), so as to follow the desired electrode overlap. In this case, we place the maximum value of the sine function in the middle of the CMR.

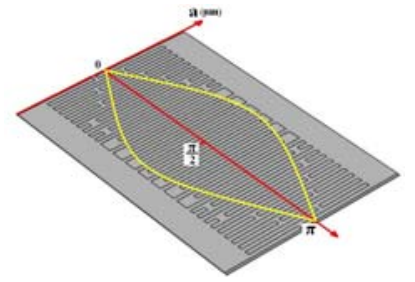

Figure 5. Schematic illustration of the sine shape, the maximum overlap (a) is placed in the middle of the CMR.

For instance, for a 13 fingers apodized CMR, we make the total device width proportional to half period of a sine wave and divide into 14 parts (Fig.6). We evaluate the sine function for each portion comprised between 0 and $\pi$, and design the finger length to be proportional to the value of the sine function at each point along the width.. Therefore, for an apodized 13 fingers CMR that uses the sine wave window, the vector representing the impulse response becomes:

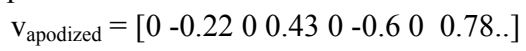

The frequency response (FR) related to the impulse response of the vector in Eq. (1) can be found by a Fourier transform (Fig. 6). As shown in Fig.6, it is clear that the CMR response is characterized by a main lobe centered around the resonance frequency and smaller out-of-band lobes. The reduction in magnitude of the side lobes is proportionally higher than the response at the center frequency. In conclusion, we can affirm that, for the 13 fingers CMR, the application of the sine-function to the shape of the electrodes reduces the spurious vibrations, represented by "smaller secondary lobes", but comes with a drop of the signal amplitude. The signal loss can be recovered by increasing the number of fingers.

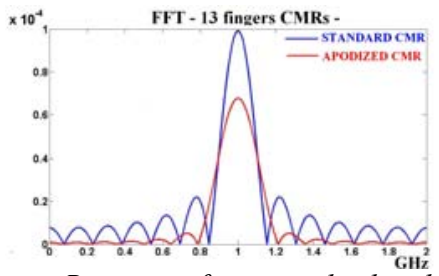

Figure 6. Frequency Response for a standard and apodized CMR.

\section{APODIZED RESONATOR DESIGN}

Beyond the suppression of spurious vibrations, the design of the apodized resonators is driven by the following guidelines: (i) operate at a center frequency around $900 \mathrm{MHz}$, (ii) attain a value of device static capacitance, $\mathrm{C}_{0}$, as compatible as possible with 50 $\Omega$ circuits; (iii) achieve quality factor, $\mathrm{Q}$ higher than 1000 in air and electromechanical coupling coefficient, $k_{t}^{2}$, higher than $1 \%$.

The overall finger width (electroded + unelectroded area) is determined by the frequency of operation. An electrode pitch of $4 \mu \mathrm{m}$ was used to operate around $900 \mathrm{MHz}$ for the given film stack. The remainder of the device geometry is set by the number of electrode pairs, their lengths and the film thickness. These parameters are used to set the device static capacitance. We selected to use an AIN film thickness of $500 \mathrm{~nm}$, finger lengths ranging between 40 and $85 \mu \mathrm{m}$, and a number of fingers between 25 and 65 to yield device capacitance between 180 and $900 \mathrm{fF}$.

The electrode design is mainly constrained by the: (i) electrode coverage ratio, which represents the ratio between the electroded area of the resonator and its overall surface; (ii) finger overlap, which, as shown in Fig. 5, represents the portion of the length of adjacent fingers that are actually overlapping. The coverage ratio was set to $50 \%$ primarily because of fabrication constraints. The electrode overlap was selected to follow, among various available window functions, the sine shape, which represents an optimal compromise to achieve spurious suppression and high coupling. In order to improve the electrical response of the resonator, we have slightly deviated from a perfect sine-shape design and modified the electrode layout in the following way: increase the overlap electrode length at the two ends of the CMR and decrease the overlap electrode length for the central fingers so as to compromise between spurious mode suppression and admittance peak reduction.

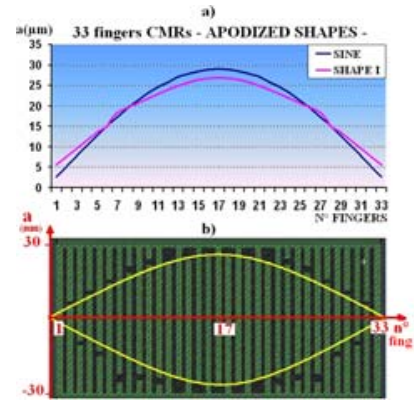

Figure 7. (a) Plot of $1 / 2$ of the finger overlap magnitude (in $\mu m$ ) for the ideal sine shape window and the selected apodization shape I (b), Layout view of the apodized CMR. 
The rationale for this selection was confirmed by COMSOL 3D analysis (Fig. 8), which shows that spurious mode suppression is attained without significantly impacting the series resonance magnitude. Shape I was effectively optimized by an empirical approach based on intuition and COMSOL $3 \mathrm{D}$ analysis. It is evident that the overlap electrode length at the end parts of the CMRs plays a key role, and a slight increase of it permits to obtain best device performance.

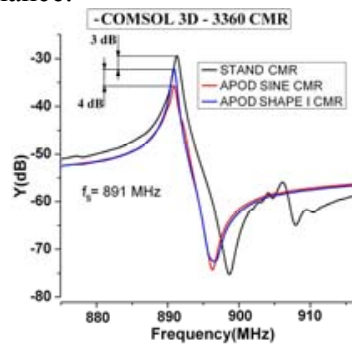

Figure 8. Admittance plot(Y) for different types of $900 \mathrm{MHz}$ AlN resonator (standard, apodized using a sine-wave window and the shape I). Y plot for a standard resonator is characterized by spurious vibrations between the series and parallel resonance. The apodized devices show suppression of the spurious modes and shape I mitigates the reduction in magnitude of admittance.

With the purpose of further improving the device electrical response, a second apodized shape (shape II) was also studied theoretically and experimentally. In shape II, the overlap length towards the ends of the resonator is further increased and made constant for the last group of fingers. The overall electrode layout is similar to shape I, except for the end parts (Fig. 9).

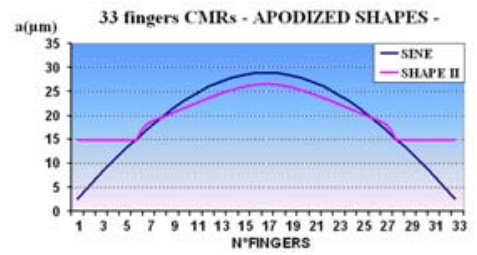

Figure 9. Plot of the finger overlap length in the case of a standard sine wave window and the selected shape II apodization scheme.

Although intuitively this design should strengthen the resonator response at the series resonance, no significant advantages were attained with respect to shape I.

\section{FABRICATION}

The two presented apodization techniques (shape I and shape II) were studied and applied (Fig. 12) to one port resonators formed by patterned top electrodes $(25,33,49$ and 65 fingers) made out of Aluminium (120 and $220 \mathrm{~nm}$ thick), and a floating bottom electrode made out of Platinum ( $90 \mathrm{~nm}$ thick) sandwiching the AlN film $(1 \mu \mathrm{m}$ thick). For comparison, the same identical geometries were laid out and fabricated without apodization (standard resonator).

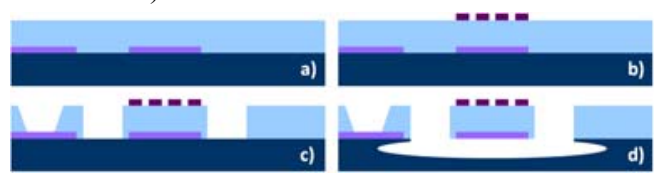

Figure 10. Representation of the 3 mask fabrication process used for the making of the apodized resonator: a) Sputtering and patterning by lift-off of the bottom electrode and sputtering of the thin-AlN film; b) deposition and patterning of the top electrode; c)Etching of AlN; d) Release of AlN resonator in $\mathrm{XeF}_{2}$ atmosphere.
The device fabrication is based on the standard 3-mask manufacturing steps previously reported for AIN CMRs [6] and here schematically reported in Fig. 10. a)

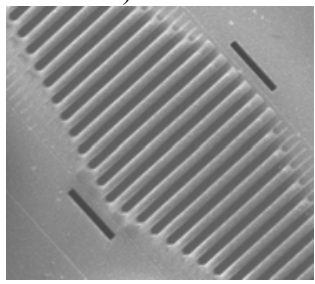

b)

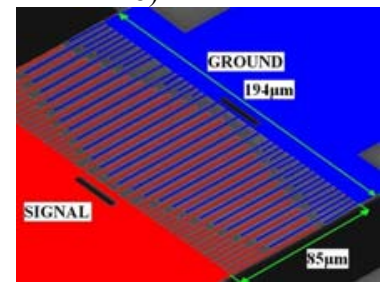

Figure 11. SEM of apodized CMR; with original colours (a), and false colours in order to highlight the apodized shape (b).

\section{EXPERIMENTAL RESULTS}

The devices were tested in a micromanipulated RF probe station under ambient conditions. A short open and load calibration was performed prior to measuring the devices and extracting their admittance response. The apodized device response is compared to standard CMR devices. For each device, admittance peak, electromechanical coupling coefficient and Q were extracted and compared. Table 1 summarizes the results for the two apodization shapes applied to $900 \mathrm{MHz}$ AlN devices. The results are presented as percentage change with respect to the non-apodized case.

Table 1 shows clearly that spurious vibrations are significantly suppressed with a minimal impact on device $Q, k_{t}^{2}$ and admittance peak value. The small value of the standard deviation $(\sigma)$ in the presented data is a sign that this technique can be applied to a large group of devices of different dimensions (and therefore different impedance). Overall, we can state that the introduction of apodization is advantageous and has mostly the following impact on the device performance: (i) complete suppression of the spurious modes; (ii) improvement of the quality factor, likely due to the ability of apodization to focus energy on the main resonance peak; (iii) minimal decrease of the electromechanical coupling coefficient $\left(\mathrm{k}_{\mathrm{t}}^{2}\right)$ and of $\mathrm{C}_{0}$; (iv) increase in the motional resistance $\left(\mathrm{R}_{\mathrm{m}}\right)$ and the consequent decrease of admittance peak (Y).
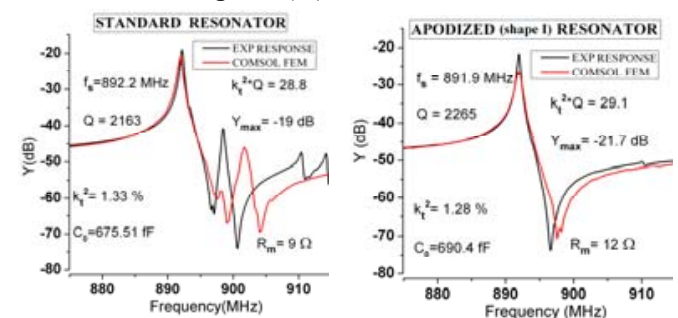

Figure 12. Experimental results for standard and apodized (shape I) $900 \mathrm{MHz}$ AlN CMR formed by $500 \mathrm{~nm}$ of AlN, $120 \mathrm{~nm}$ of Al, 90 $\mathrm{nm}$ of Pt and 49 fingers (finger length of $85 \mu \mathrm{m}$ ).

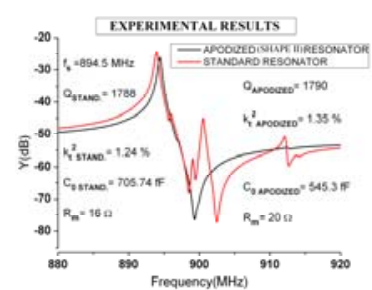

Figure 13. Experimental results for standard and apodized (shape II) $900 \mathrm{MHz}$ AlN CMR formed by $500 \mathrm{~nm}$ of AlN, $120 \mathrm{~nm}$ of Al,90 $\mathrm{nm}$ of Pt, and 33 fingers (finger length of $85 \mu \mathrm{m}$ ). 
It is worth noting that the experimental results are in line with the theoretical expectations as shown in the comparison between the experimental response and the COMSOL FEM model (Fig. 12). The same analysis performed for shape I, was also conducted for shape II (Fig. 13).

From the experimental results, it is evident that, also in this case, the device response for a standard resonator is characterized by large spurious between the series and parallel resonance, while an apodized resonator (shape II) shows complete suppression of the spurious vibrations.

Table 1: Summary of the results for a variety of apodized resonators (shape I and II) operating around $900 \mathrm{MHz}$ and formed by $500 \mathrm{~nm}$ of AlN, $120 \mathrm{~nm}$ of Al and $120 \mathrm{~nm}$ of Pt. The resonators are identified with a code of four numbers; the first two numbers indicate the number of fingers and the last two refer to the length of fingers in $\mu \mathrm{m}$. So, for instance the code "2560" is referred to a resonator of 25 fingers, with a length of $60 \mu \mathrm{m}$. The data are provided as percentage change with respect to the linear value of the non-apodized case. A negative value of $\%$ indicates that a higher value is recorded in the non-apodized case. The decrease of spurious vibrations has been quantified by evaluating the maximum amplitude of the spurious response in the admittance graph (expressed in linear values) before $\left(S p_{\text {stand }}\right)$ and after the application of the apodization technique $\left(S p_{\text {apod }}\right)$. The same concept has been applied in order to calculate the decrease of quality factor $(\Delta Q)$, electromechanical coupling coefficient $\left(\Delta k_{t}^{2}\right)$, and of the admittance peak ( $\triangle Y)$. So, for $\triangle S p=-100 \%$, it means that a total suppression of the spurious vibration has been achieved.

$$
\Delta S p=\frac{S p_{a p}-S p_{s \tan d}}{S p_{s \tan d}} * 100
$$

\begin{tabular}{|c|c|c|c|c|c|c|c|c|}
\hline \multicolumn{5}{|c|}{ Apodization I } & \multicolumn{4}{|c|}{ Apodization II } \\
\hline CMRs & $\Delta \mathrm{Sp}$ & $\Delta \mathbf{Q}$ & $\Delta \mathrm{Y}$ & $\Delta \mathbf{k}_{\mathrm{t}}^{2}$ & $\Delta \mathbf{S p}$ & $\Delta \mathbf{Q}$ & $\Delta \mathbf{Y}$ & $\Delta \mathbf{k}_{\mathrm{t}}^{2}$ \\
\hline 2540 & $-69.3 \%$ & $6.7 \%$ & $-6.6 \%$ & $-12.1 \%$ & $-79.3 \%$ & $-11.4 \%$ & $-11.9 \%$ & $-18.9 \%$ \\
\hline 2560 & $-94.0 \%$ & $-2.8 \%$ & $-13.5 \%$ & $-7.1 \%$ & $-95.2 \%$ & $-2.9 \%$ & $-20.4 \%$ & $3.2 \%$ \\
\hline 2585 & $-100.0 \%$ & $-42.1 \%$ & $-27.2 \%$ & $10.2 \%$ & $-100.0 \%$ & $-35.5 \%$ & $-25.6 \%$ & $-1.6 \%$ \\
\hline 3340 & $-62.1 \%$ & $-15.8 \%$ & $-8.9 \%$ & $-13.3 \%$ & $-51.2 \%$ & $-15.7 \%$ & $-10.6 \%$ & $-14.8 \%$ \\
\hline 3360 & $-94.7 \%$ & $-2.8 \%$ & $-13.4 \%$ & $-6.4 \%$ & $-97.9 \%$ & $-6.7 \%$ & $-8.8 \%$ & $6.4 \%$ \\
\hline 3385 & $-100.0 \%$ & $11.1 \%$ & $-10.7 \%$ & $-6.3 \%$ & $-100.0 \%$ & $0.1 \%$ & $-7.4 \%$ & $6.3 \%$ \\
\hline 4940 & $-97.4 \%$ & $-12.6 \%$ & $-15.5 \%$ & $-24.8 \%$ & $-97.4 \%$ & $-32.8 \%$ & $-20.3 \%$ & $-25.5 \%$ \\
\hline 4960 & $-100.0 \%$ & $4.8 \%$ & $-12.3 \%$ & $-12.0 \%$ & $-100.0 \%$ & $4.8 \%$ & $-18.0 \%$ & $-15.5 \%$ \\
\hline 4985 & $-100.0 \%$ & $4.7 \%$ & $-14.2 \%$ & $-8.5 \%$ & $-100.0 \%$ & $4.8 \%$ & $-17.4 \%$ & $-12.7 \%$ \\
\hline 6540 & $-78.8 \%$ & $-7.2 \%$ & $-24.3 \%$ & $-25.0 \%$ & -- & -- & -- & -- \\
\hline 6560 & $-100.0 \%$ & $10.0 \%$ & $-19.9 \%$ & $-31.0 \%$ & -- & -- & -- & -- \\
\hline 6585 & $-100.0 \%$ & $-4.0 \%$ & $-18.0 \%$ & $-27.8 \%$ & -- & -- & -- & -- \\
\hline$\mu$ & $-91.4 \%$ & $-4.2 \%$ & $-15.4 \%$ & $-13.7 \%$ & $-91.2 \%$ & $-10.6 \%$ & $-15.6 \%$ & $-8.1 \%$ \\
\hline$\sigma$ & $13.3 \%$ & $14.7 \%$ & $6.1 \%$ & $11.7 \%$ & $16.4 \%$ & $15.0 \%$ & $6.2 \%$ & $11.9 \%$ \\
\hline
\end{tabular}

Table 2: Summary of performances for a variety of apodized resonators (shape I and II) operating around $900 \mathrm{MHz}$ and formed by $500 \mathrm{~nm}$ of AlN, $90 \mathrm{~nm}$ of Pt and the Al top electrodes of $220 \mathrm{~nm}$.

\begin{tabular}{|c|c|c|c|c|c|c|c|c|}
\hline \multicolumn{9}{|c|}{$120 \mathrm{~nm}$ AI TOP ELECTRODES } \\
\hline & \multicolumn{4}{|c|}{ Apodization I } & \multicolumn{4}{|c|}{ Apodization II } \\
\hline & $\mathbf{Q}$ & $\mathbf{k}_{t}^{2}$ & $\mathbf{Y}(\mathrm{dB})$ & $\mathrm{C}_{0}(\mathrm{fF})$ & $\mathbf{Q}$ & $\mathbf{k}_{\mathrm{t}}^{2}$ & $\mathrm{Y}(\mathrm{dB})$ & $\mathrm{C}_{0}(\mathrm{fF})$ \\
\hline $\boldsymbol{\mu}$ & 1250.1 & $1.55 \%$ & -32.82 & 418.6 & 1305.2 & $1.27 \%$ & -32.2 & 367.11 \\
\hline $\boldsymbol{\sigma}$ & 423.4 & $0.11 \%$ & 3.51 & 188.7 & 267.7 & $0.12 \%$ & 3.52 & 140.8 \\
\hline \multicolumn{9}{|c|}{$220 \mathrm{~nm}$ Al TOP ELECTRODES } \\
\hline & \multicolumn{4}{|c|}{ Apodization I } & \multicolumn{4}{|c|}{ Apodization II } \\
\hline & $\mathbf{Q}$ & $\mathbf{k}_{\mathrm{t}}{ }^{2}$ & $\mathbf{Y}(\mathbf{d B})$ & $C_{0}(f F)$ & $\mathbf{Q}$ & $\mathbf{k}_{\mathrm{t}}{ }^{2}$ & $\mathbf{Y}(\mathrm{dB})$ & $C_{0}(f F)$ \\
\hline$\mu$ & 1848.8 & $1.14 \%$ & -27.9 & 454.9 & 1733.1 & $1.22 \%$ & -29.7 & 376.3 \\
\hline$\sigma$ & 367.3 & $0.16 \%$ & 4.95 & 200.3 & 409.3 & $0.10 \%$ & 4.75 & 156.6 \\
\hline
\end{tabular}

Moreover, we verified that the apodized shapes can be successfully applied to CMRs characterized by different thicknesses of the Al top electrode $(120$ and $220 \mathrm{~nm})$. Table 2 reports the statistical performances of samples of the apodized CMRs characterized by $120 \mathrm{~nm}$ and $220 \mathrm{~nm} \mathrm{Al}$ top electrodes. Table 3 shows how apodized CMRs characterized by different top electrode thicknesses present similar performances in terms of spurs reduction, $\Delta \mathrm{Q}, \Delta \mathrm{k}_{\mathrm{t}}^{2}$ and $\Delta \mathrm{Y}$. These data further validate the broad applicability of this apodization technique.

Table 3. Summary of results for a variety of apodized resonators (shape I and II) operating around $900 \mathrm{MHz}$; formed by $500 \mathrm{~nm}$ of AlN, $90 \mathrm{~nm}$ of Pt and the Al top electrodes of $120 \mathrm{~nm}$ and $220 \mathrm{~nm}$.

\begin{tabular}{|c|c|c|c|c|c|c|c|c|}
\hline \multicolumn{9}{|c|}{$120 \mathrm{~nm} \mathrm{Al} \mathrm{TOP} \mathrm{ELECTRODES}$} \\
\hline & \multicolumn{4}{|c|}{ Apodization I } & \multicolumn{4}{|c|}{ Apodization II } \\
\hline & $\Delta \mathrm{Sp}$ & $\Delta \mathbf{Q}$ & $\Delta \mathbf{Y}$ & $\Delta \mathbf{k}_{\mathrm{t}}^{2}$ & $\Delta \mathrm{Sp}$ & $\Delta \mathbf{Q}$ & $\Delta \mathbf{Y}$ & $\Delta \mathbf{k}_{t}^{2}$ \\
\hline$\mu$ & $-98.9 \%$ & $3.7 \%$ & $-28.7 \%$ & $-19.9 \%$ & $-95.8 \%$ & $9.3 \%$ & $-24.7 \%$ & $-16.3 \%$ \\
\hline$\sigma$ & $2.52 \%$ & $21.61 \%$ & $14.89 \%$ & $12.69 \%$ & $5.6 \%$ & $14.8 \%$ & $18.6 \%$ & $-13.2 \%$ \\
\hline \multicolumn{9}{|c|}{$220 \mathrm{~nm}$ Al TOP ELECTRODES } \\
\hline & \multicolumn{4}{|c|}{ Apodization I } & \multicolumn{4}{|c|}{ Apodization II } \\
\hline & $\Delta \mathrm{Sp}$ & $\Delta \mathbf{Q}$ & $\Delta \mathbf{Y}$ & $\Delta \mathbf{k}_{t}^{2}$ & $\Delta \mathrm{Sp}$ & $\Delta \mathbf{Q}$ & $\Delta \mathbf{Y}$ & $\Delta \mathbf{k}_{t}^{2}$ \\
\hline$\mu$ & $-91.4 \%$ & $-4.2 \%$ & $-15.4 \%$ & $-13.7 \%$ & $-91.2 \%$ & $-10.6 \%$ & $-15.6 \%$ & $-8.1 \%$ \\
\hline$\sigma$ & $13.3 \%$ & $14.7 \%$ & $6.1 \%$ & $11.7 \%$ & $16.4 \%$ & $15.0 \%$ & $6.2 \%$ & $11.9 \%$ \\
\hline
\end{tabular}

\section{CONCLUSIONS}

This paper reported on the application of apodization techniques to $900 \mathrm{MHz}$ MEMS AlN Contour-Mode Resonators (CMRs) to efficiently suppress spurious modes in close proximity of the main mechanical resonance without impacting their $Q$ or $k_{t}^{2}$. The obtained results show that the introduced apodization techniques, until now exclusively applied to SAW devices, have a significant potential for CMRs and could potentially be extended to several other MEMS resonator technologies.

\section{AKNOWLEDGEMENTS}

Travel support has been generously provided by the Transducer Research Foundation. Funding for this project was provided through the DARPA M/NEMS S\&T program.

\section{REFERENCES}

[1] G. Piazza, et al., "Piezoelectric Aluminum Nitride Vibrating Contour- Mode MEMS Resonators", J.MEMS, vol.15, p. 1406-1418, 2006

[2] B.P. Harrignton, et al., "Toward ultimate performance in $\mathrm{GHz}$ MEMS Resonators: low impedance and high Q", J.MEMS, 2010 IEEE 23rd International Conference, p. 707-710, 2010

[3] Y. Xie, et al., "1.52-GHz Micromechanical Extensional WineGlass Mode Ring Resonators", J.MEMS, Ultrasonics, Ferroelectrics and Frequency Control, IEEE Transactions on, vol.55, p. 890-907, 2008

[4] Olsson, et al., "Origins and Mitigation of Spurious Modes in AIN Microresonators", Proc. of the IEEE International Ultrasonics Symposium, p.1272-1276, October 2010

[5] C.S. Hartmann, D.T. Bell, R.C., Rosenfeld, "Impulse Model Design of Acoustic. Surface-Wave Filters", IEEE Transactions on Sonics nd Ultrasonics, vol.20, p. 80-93, 1973

[6] M. Rinaldi, C. Zuniga, C. Zuo, and G. Piazza, "Super-HighFrequency Two-Port AlN Contour-Mode Resonators for RF Applications" IEEE Transactions on Ultrasonics, Ferroelectrics, and Frequency Control, vol. 57, no. 1, 2010.

\section{CONTACTS}

M. Giovannini, Politecnico of Milan (marco.giova23@gmail.com); G. Piazza,CMU (piazza@ece.cmu.edu). 